
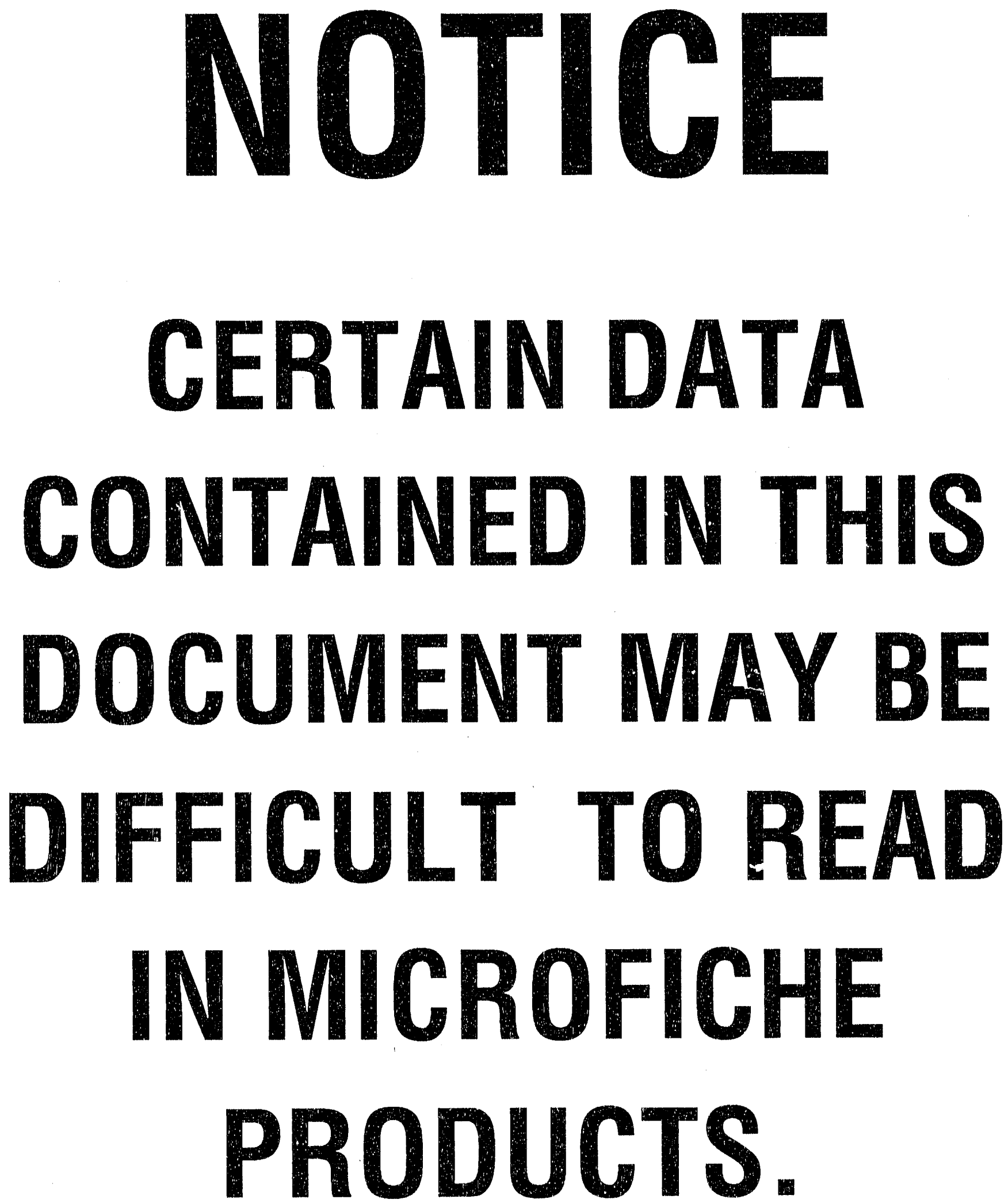
TRANSIENT STRESS EVOLUTION AND

CRYSTALLIZATION IN LASER-IRRADIATED

AMORPHOUS TITANIA SOL-GEL FILMS

\author{
G. J. Exarhos \\ N. J. Hess \\ S. Wood
}

October 1991

\author{
Presented at the \\ Symposium on Cotical Materials for High \\ Power Lasers \\ October 23-25, 1991 \\ Boulder, Colorado
}

Work supported by

the U.S. Department of Energy

under Contract DE-ACO6-76RLO 1830

\author{
Pacific Northwest Laboratory \\ Richland, Washington 99352
}

\title{
DISCLAIMER
}

This report was prepared as an account of work sponsored by an agency of the United States Government. Neither the United States Government nor any agency thereof, nor any of their employees, makes any warranty, express or implied, or assumes any legal liability or responsibility for the accuracy, completeness, or usefulness of any information, apparatus, product, or process disclosed, or represents that its use would not infringe privately owned rights. Reference herein to any specific cummercial product, process, or service by trade name, trademark, manufacturer, or otherwise does not necessarily constitute or imply its endorsement, recommendation, or favoring by the United States Government or any agency thereof. The views and opinions of authors expressed herein do not necessarily state or reflect those of the United States Government or any agency thereof.

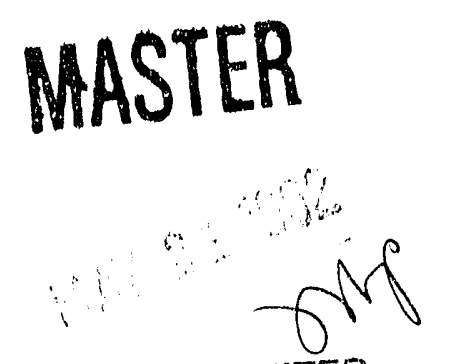


Transient stress evolution and crostallizalion

in laser-irradiated amorphous titania sol-gel films

\author{
Gregory J. Exarhos and Nancy J. Hess \\ Pucific Northwest Laboratory \\ PO BOX 099. MS K2-44 \\ Richland, WA 99352 \\ Susanne Wood \\ Department of Physics \\ Shock Dynamics Laboratory \\ Washington State University \\ Pullman, WA 99164-2814
}

\begin{abstract}
Amorphous $\mathrm{TiO}_{2}$ soi-gel films are irreversibly transformed to a crystalline anatase phase when heated to temperatures in excess of $575 \mathrm{~K}$ or subjected to intense pulsed or $\mathrm{CW}$ laser irradiation. The laser-induced transformation is initiated as a result of impurity absorption and subsequent heating, and results in densification and relative changes in compressive stress of the film. Isothermally annealed films exhibit a decrease in compressive stress as crystallization proceeds while an increase in compressive stress followed by a decrease in stress is observed when crystallization is laser-induced. Raman spectroscopy has been used to characterize the crystallization ingrowth kinetics and is used in this work as a real time probe of both film temperature and localized stress which can be evaluated from shifts in lattice phonon frequencies measured in real time during laser irradiation. The laser not only induces the phase transformation but excites inelastic Raman scattering from which film stress and temperature can be estimated. A second approach for the determination of these parameters requires incorporation of a thin ruby film between the titania and silica substrate. Here, the wavelength shift of the laser-induced ruby fluorescence can be used to quantify interfacial stress; the fluorescence lifetime measurements are used to determin: temperature. The advantages and limitations of these techniques for evaluating transient stress and temperature evolution in thin titania films subjected to CW laser irradiation will be discussed.
\end{abstract}

\title{
1. INTRODUCTION
}

Titanium dioxide $\left(\mathrm{TiO}_{2}\right)$ films can be deposited onto glass or metal substrates readily from solutions containing organometallic ester precursors using spin casting or dip-coating procedures. Such films exhibit refractive indices markedly lower than single crystal values owing to the amorphous nature of the film and the void microstructure which evolves as the film densifies. Such films are observed to irreversibly' crystallize when heated to temperatures above $575 \mathrm{~K}$, or during exposure to high energy pulsed or CW laser irradiation. $[1,2,3]$ Energy absorption by impurities present in the film, and the film microstructure which creates microscopic variations of field intensit: upon high energy' laser irradiation are but two causes for localized heating. [4] The dynamics of the cry'stallization process are influenced by the film microstructure and the magnitude of stress inherent in the cast film and that which develops as the film is heated. Time-resolved laser Raman spectroscopy is used in this work to follow in real time the kinetics of the film crystallization phenomenon, to determine film temperature, and to evaluate changes in film stress during the 
transformation. Results reported in this paper reveal contrasting behavior in the film stress which evolves in free-standing titania films and films deposited on silica substrates.

Raman techniques have been used extensively to evaluate haser induced damage to single and multilayer titania films. Recent work in this area and a sumbry of previous work appear in the paper by White, ot al, 15] where the utility of inelastic light scattering measurements for characterization of crystalline phase and residual stress in laser damaged films has been demonstrated. The overall appearance of the vibrational spectrum serves to identify a particular crystalline phase, while slight increases in phonon frequencies can be correlated with residual compressive stress in a thin film. [3] Most of this work has been performed on films at room temperature both before and after high energy pulsed laser irradiation.

Raman techniques also have been used to study in real time the isothermal crystallization kinetics of amorphous sol-gel titania films. [6] During this process, the volume fraction of the evolved spheroidal crystalline phase can be correlated with the Raman intensity of the strongest observed vibrational mode. Analysis of the sigmoidal crystallite ingrowth curves in terms of a moditied Avrami model provides a rate constant for the process as well as a critical exponent which is related to the morphology of crystal growth. A review of this data revealed that mode frequencies also were perturbed during the course of crystallization suggesting a concomitant change in residual film stress. This observation suggested that time resolved Raman methods could be used to evaluate. changes in residual film stress during laser-induced crystallization of these and similar films. Therefore, two methods based upon this previous work $[7,8]$ have been developed for obtaining simultaneous stress/temperature information during the crystallization process. The following sections review the principles behind these techniques and discusses the limitations and precision of measured data.

\subsection{RESIDUAL STRESS/TEMPERATURE DETERMINATION IN FTLMS}

\subsection{Raman measurements of anatase in a diamond anvil cell $(P, T)$ \{Contour Method}

The expansion/contraction of a crystal lattice with applied temperature/pressure results from anharmonicity in the lattice potential energy. A related dependence of phonon frequencies on temperature and pressure also is observed. [9] Therefore, it appears possible that sample pressur: and temperature can be extracted from vibrational frequency measurements. Fig. la depicts a vibrational frequency surface in pressure-temperature space for the high frequency $E_{\text {, mode of the }}$ anatase crystalline phase of $\mathrm{TiO}_{2}$. The curve was constructed on the basis of eqn. 1 , which is an empirical analytical representation of the pressure and temperature dependence of the mode frequency determined by Raman measurements of single crystal $\mathrm{TiO}_{2}$ in a diamond anvil cell.

$$
\omega_{i}=\omega_{i}^{0}+a_{i} P+b_{i}^{T}+c_{i}^{P T} \text {. }
$$

Here $\omega_{i}$ is the frequency of the mode of interest, and $a_{j}, b_{i}$ and $c_{i}$ are fitting parameters. The intersection of a horizontal plane, constructed at a particular frequency, with this surface defines? curve of constant frequency. There are a number of combinations of $T$ and $P$ which cxist for this fixed frequency. Therefore, measurement of a single vibrational frequency is not sufficient to extract this information. However, measurement of a second vibrational mode frequency which exhibits , different $P, T$ dependence would allow sample pressure and temperature to be determined uniquely 
Fig. 1b shows the frequency surface in P,T space for the low frequency $E_{g}$ mode of analase. Notice that in contrast to the $6.36 \mathrm{~cm}^{-1}$ mode, the frequency increases as both temperature and pressure are increased. A contour construction of curves of equal frequency in P,T space for both these modes appears in Fig. 1c. The crossing points of these curves identify specific pressures and temperatures for the measured frequencies of the two independent vibrational modes. A numerical solution of eqn. 1 for the two frequencies of interest can be used to extrac: the required P, T information. The calculated pressure is related to the compressive stress felt by the sample.

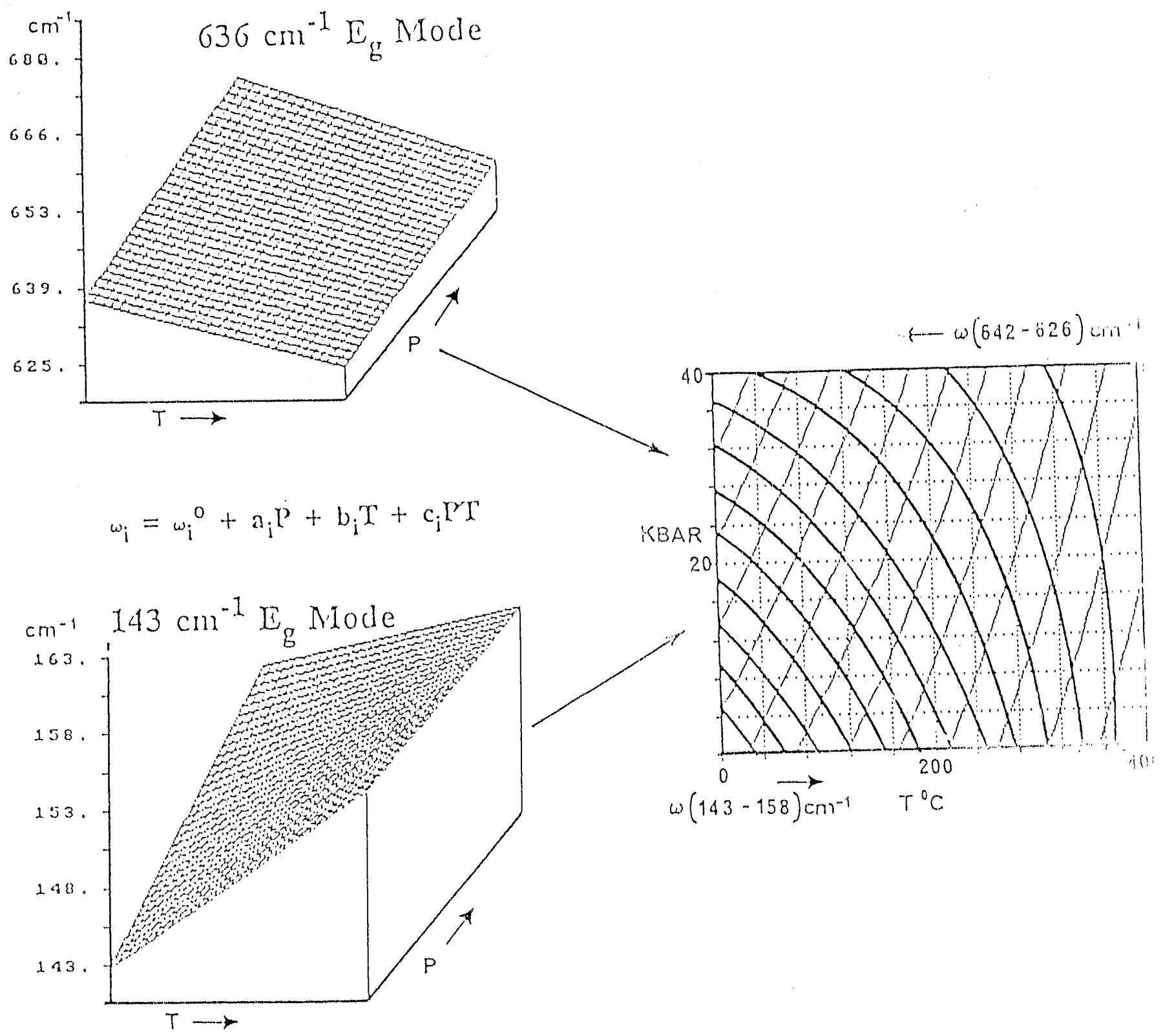

Fig. 1. Protocol for extracting sample temperalure and compressive stress information from vibrational mode frequenc: measurements. (a) The vibrational frequency surface in P,T space for the $6.36 \mathrm{~cm}^{-1}$ mode. (b) The vibrational frequency surface for the $143 \mathrm{~cm}^{-1}$ mode. (c) Contour plot showing curves of equal frequency for the two mortes, ia $P, T$ space. (Bold lines are for the low frequency mode, dashed lines are for the high frequency morle. All rit." ". reported for single crystal $\mathrm{TiO}_{2}$ (annatase) in a heated Diamond Anvil Cell. 
This method works well provided that the P,T response of the wo frequencies selected are well known and not of the same magnitude. Should these conditions not be met the location of the crossing points will be subject to increased uncertainty.

\subsection{Fluorescence measurements of an interfacial probe ion}

A second non-intrusive method for determination of sample temperatures and interfacial stress in thin films is based upon variations in the iluorescence response of a probe ion introduced at the film substrate interface. Ruby $\left(\mathrm{Cr}^{+3}: \mathrm{Al}_{2} \mathrm{O}_{3}\right)$ exhibits an intense red fluorescence under blue-green excitation. The fluorescence wavelength is known to depend strongly on both temperature and pressure. However, the fluorescence lifetime of ruby has been shown to decrease markedly with increasing temperature and is relatively pressure independent under the relative stress conditions encountered here. [10] Therefore, measurements of the fluorescence wavelength and liferime are. sufficient to determine the ambient temperature and pressure. The introduction of a thin ruby film between a silica substrate and titania overlayer should serve as an interfacial probe of both temperature and stress. The variation of the fluorescence wavelength and lifetime with pressure and.l temperature (514.5 $\mathrm{nm}$ excitation) are shown in Fig.2 for a thin ruby film deposited on silica.
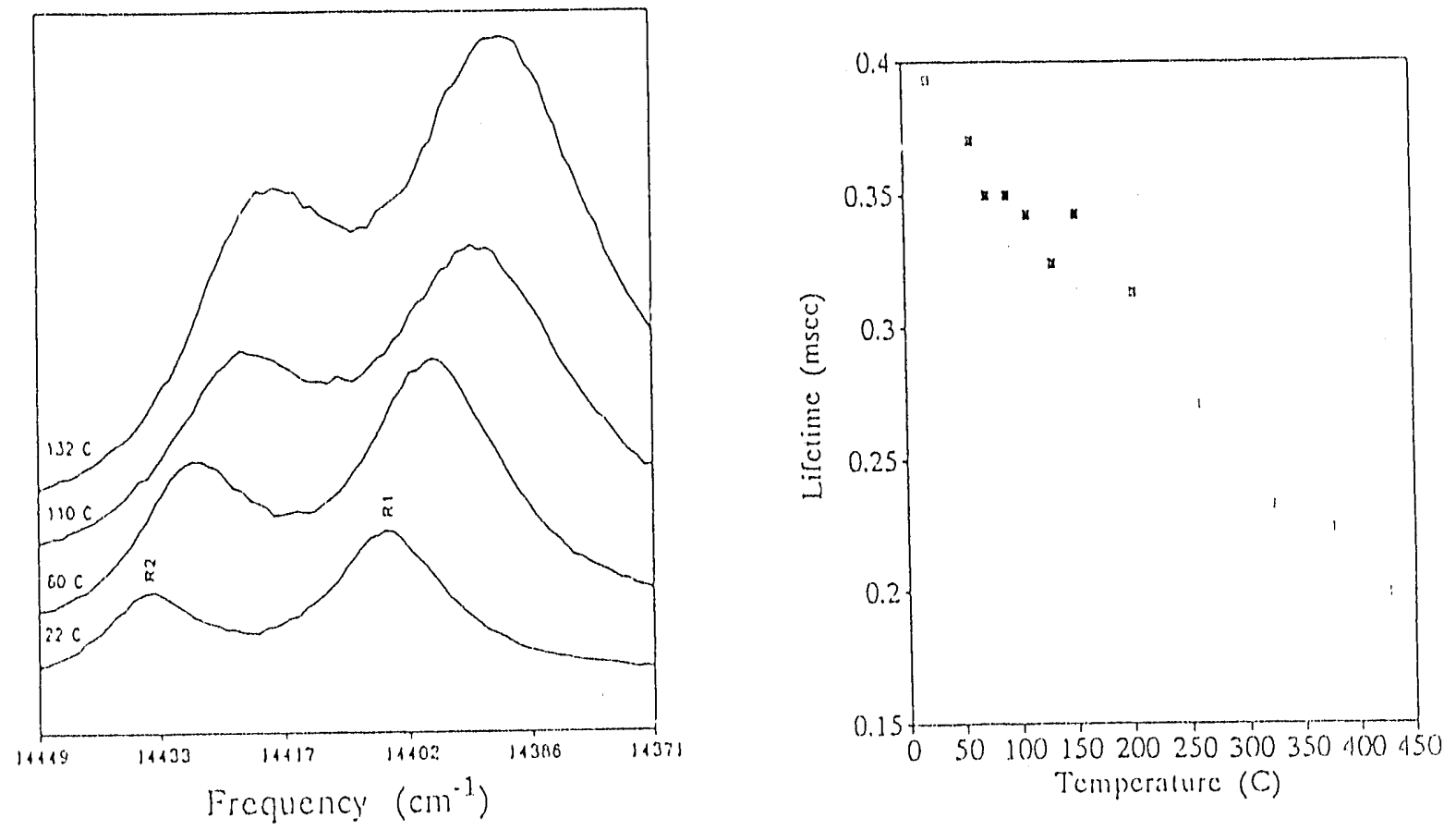

Fig 2. Variation in fluorescence wavelength and lifetime for a $300 \mathrm{~nm}$ ruby film on silica irradiated by $20 \mathrm{ml}$, 514.5 num radiation.

\subsection{EXPERIMENTAL}

Sol-gel titania films were prepared from highly acidic stock solutions. The details of the solution preparation are given elsewhere [6,11]. Films were deposited onto silica substrates using spin. casting techniques at rates between 1200 to $1700 \mathrm{rpm}$ for 60 seconds. Uniform films resulted which were approximately 200 to $600 \mathrm{~nm}$ thick as determined from transmission spectroscopy. The thickr.i films tend to crack after spin-casting and small fragments can be removed from the silica subsim. 
A sol-gel precursor solution also was used to prepare the ruby interfacial layer. A concentrated acidified stock solution was prepared using aluminum isopropoxide and ethanol. Even with the high acid content of these solutions, precipitates tended to form if the solution stood for longer than a week. Surface preparation of silica substrates involved etching by alternate baths of concentrated $\mathrm{HCl}$ and $\mathrm{NH}_{4} \mathrm{OH}$ followed by a thorough rinse with distilled $\mathrm{H}_{2} \mathrm{O}$. The ruby films were deposited onto the prepared silica substrates by spin-casting methods at $8000 \mathrm{rpm}$ for 60 seconds. The resulting amorphous film was crystallized to the a-A $\mathrm{A}_{2} \mathrm{O}_{3}$ structure by placing the conted substrate in a furnace pre-heated $101400^{\circ} \mathrm{C}$ for 10 minutes followed by a rapid quench to rom temperature. The $\alpha-\mathrm{Al}_{2} \mathrm{O}_{3}$ structure was verified by measuring the characteristic fuorescence spectrum of ruby. The intensity of the fluorescence could be increased by depositing successive multilayers. However, the resulting multilayer film appeared somewhat cloudy. Temperature dependent fluorescence lifetimes of the ruby interfacial laver were determined using a phase modulation technique [10] over the temperature range from $2010500 \mathrm{C}$ for calibration purposes. After calibration, an amorphous titania sol-gel film was deposited onto the ruby film using the technique described earlier.

Time-resolved Raman spectroscopy was used to monitor the crystallization process of the free standing amorphous titania sol-gel films and the films deposited on top of the ruby interfacial layer. For isothermal crystallization of free-standing films, a film fragment approximately $100 \mu m$ in diameter was placed into the empty cavity of a diamond anvil cell which served only as a sample holder. The cell was then lowered into a resistively-heated, proportionately controlled, copper block furnace that was pre-heated to the desired temperature. Raman scattering was excited with approximately $100 \mathrm{~mW}$ of $514.5 \mathrm{~nm}$ radiation from an argon ion laser focused by a $10 \mathrm{X}$ objective 10 a spot size approximately $10 \mu \mathrm{m}$ in diameter. The scattered light was collected using a $180^{\circ}$ backscattering geometry by the same objective and dispersed by a triple spectrometer onto a liquid nitrogen cooled charged coupled device detector consisting of an array of $1152 \times 278$ diodes. A two minute time resolution was used for these experiments which typically required several hours.

Time-resolved Raman measurements of the laser-induced crystallization of free-standing film: were performed using the sampling geometry described above; however these film fragments were. not placed in a diamond anvil cell or the copper block furnace. Crytallization of the films was initiated with approximately $75 \mathrm{~mW}$ of $488.0 \mathrm{~nm}$ radiation. The time resolution for thes: experiments was 30 seconds during the ten minute interval over which crystallization occurred.

Time-resolved Raman measurements of laser-induced crystallization in sol-gel titania films deposited on the interfacial ruby layer on silica required a $90^{\circ}$ scattering geometry. Crystallization was initiated with 2.95 to $3.15 \mathrm{~W}$ using all lines from a CW argon ion laser. The radiation was focused to a $100 \mu \mathrm{m}$ spot size at the sample. In this geometry the scatured light was collected by an elliptical mirror and directed to the triple spectrometer as before. For simultaneous collection of the ruby fluorescence a long wavelength reflecting mirror was placed above the sample. 'This mirror allowed the green light from the argon ion laser transmitted through the sample to pass but reflected the red ruby fluorescence onto a silicon photodiode. A mechanical chopper operating at $500 \mathrm{~Hz}$ was placed in the beam path. The photodiode output was synchronously detected using a lock-in amplifier which was used to measure the phase delay between the probe excitation and the fluorescent signal recorded by the diode. The time resolution for these experiments was 0.3 seconds. for the acquisition of the Raman spectra and 0.5 seconds for the ruby fluorescence phase measurement over a 10 second interval. 
The peak positions and the intensity of the observed Raman vibrational modes were determined by curvefitting the time-resolved spectra using a commercially available software package, SpectraCales. Prior to fitting, all the spectra acquired during a crystallization run were ratioed to the first spectrum to remove spectral artifacts attributed to the silica substrate. For fitting purposes, all lineshapes were assumed to be Lorentzian.

\subsection{RESUL'TS}

Raman spectra of amorphous sol-gel deposited titania films show two very weak, broad foatures at $430 \mathrm{~cm}^{-1}$ and $600 \mathrm{~cm}^{-1}$. [8] During the crystallization process these broad features are replaced by five intense, relatively sharp lines at $143 \mathrm{~cm}^{-1}, 199 \mathrm{~cm}^{-1}, 398 \mathrm{~cm}^{-1}, 513 \mathrm{~cm}^{-1}$, and $636 \mathrm{~cm}^{-1}$ that are characteristic of the crystalline anatase phase. Fig. 3 depicts a collection of time-resolved Raman spectra measured during the isothermal crystallization of a free-standing film fragment at $350^{\circ} \mathrm{C}$.

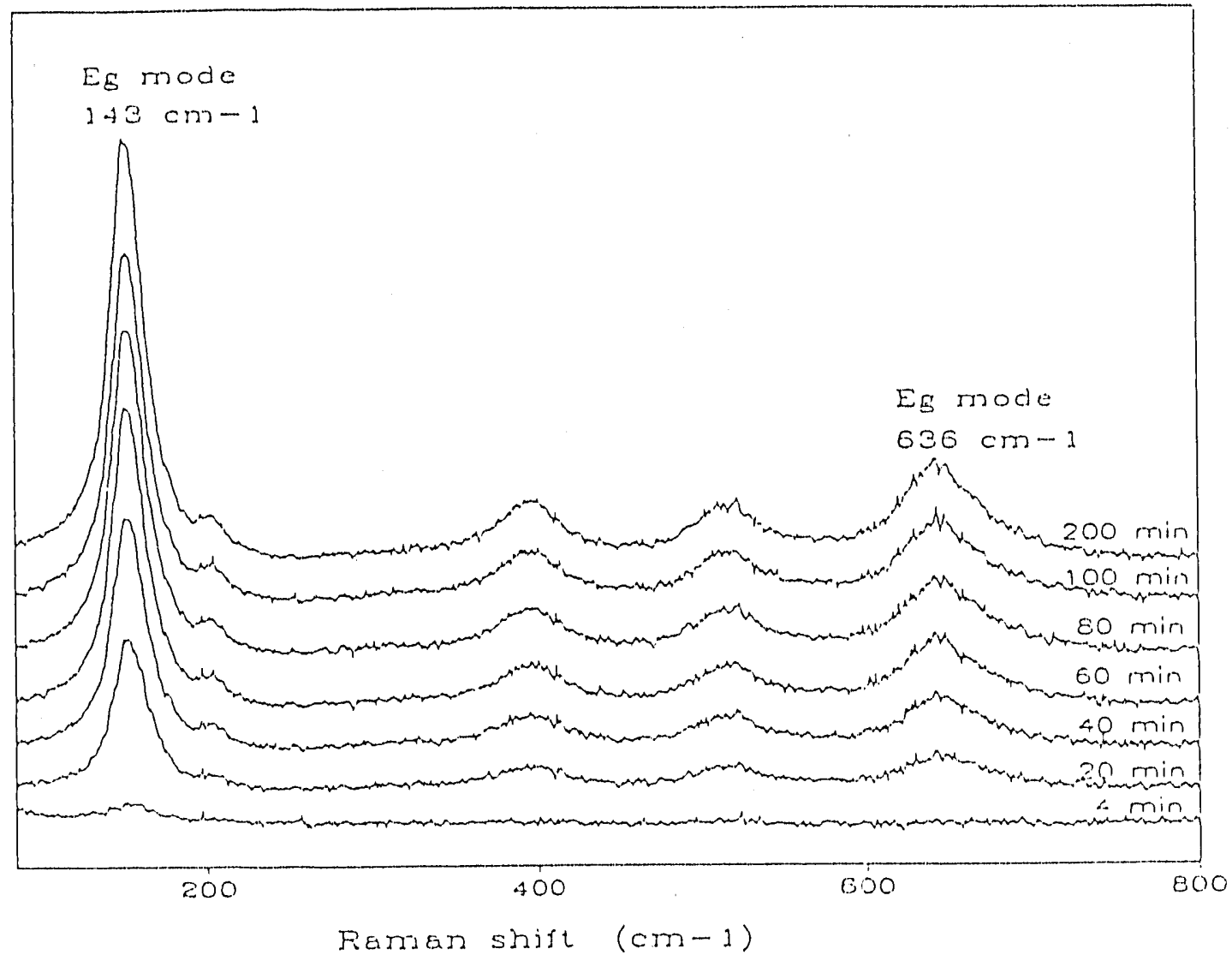

Fig 3. Raman spectra measured during isothermal crystallization of amorphous sol-gel deposited titania films.

The vibrational mode intensity correlates with the degree of crystallinity of the film. [12] Using tirue-resolved Raman spectroscopy, the intensity of the $143 \mathrm{~cm}^{-1}$ mode is used to follow the development of crystallinity in the film as a function of tine and is illustrated for an isotherma crystallization process in Fig. 3a. Similar plots are shown for the laser-induced crystallization of the free-standing film and for film attached to a substrate in Fig. 4 a and 5 a, respectively. The resulting curves have been analyzed in terms of a modified Avrami model which includes wn: diffusion effects to the nucleation center based upon the microstructure. [6] 


$$
F(1)_{T}=1-\exp \left(-\kappa_{T}, \eta^{\eta}{ }^{\eta}\right)
$$

Here $\kappa_{T, \eta}$ is the temperature-dependent crystallization rate constant and $\eta$ is the critical growth exponent. Table 1 lists the crystallization rate constants and the critical growth exponents determined for the three types of cry'stallization experiments described here.

TABLE 1

\begin{tabular}{lll}
\multicolumn{1}{c}{ Exyeriment } & $\underline{\kappa}_{\mathrm{T}, \eta}$ & $n$ \\
$\begin{array}{l}\text { isothermal crystallization } \\
\text { of frec-standing film }\end{array}$ & 0.020 & 1.02 \\
$\begin{array}{l}\text { laser-induced crystallization } \\
\text { of frec-standing film }\end{array}$ & 0.200 & 1.55 \\
$\begin{array}{l}\text { lascr-induccd crystallization } \\
\text { of film on substratic }\end{array}$ & 0.250 & 3.75
\end{tabular}

In addition to intensity data as function of time, from which the crystallization kinetics can be determined, the temperature and state of stress in the film can be extracted from the time-resolved Raman spectra. Fig. 1 illustrates the temperature and pressure dependence of the $E_{\mathrm{f}}$ modes of anatase at $143 \mathrm{~cm}^{-1}$ and $636 \mathrm{~cm}^{-1}$. Because the signs of the temperature derivative for these modes are different, the contours of the observed Raman shift as a function of pressure and temperature intersect at an high angle as shown in Fig. 1c. The intersection of these surfaces can be used to graphically determine an unique pressure and temperature if the Raman shift of two $E_{\mathrm{g}}$ modes are known. However, we have chosen to solve for pressure and temperature numerically using a short FORTRAN code. The Raman shift of the $E_{\mathrm{g}}$ modes are determined as a function of time from fitting the time-resolved Raman spectra. These frequency determinations are shown as a function of time in Fig. $3 b$ and $4 b$ for the crystallization experiments involving free-standing film fragments.

Raman intensity variations during isothermal crystallization of the free-standing film are showr1 in Fig. $4 \mathrm{a}$; the Raman shift of both $\mathrm{E}_{\mathrm{g}}$ modes decreases during the crystallization process (Fig, 4b). This in contrast to the behavior observed during laser-induced crystallization of the free-standing film shown in Fig. 5b. At the onset of crystallization, the frequency of the $143 \mathrm{~cm}^{-1}$ mode increases and then decreases as the crystallization process nears completion. The frequency of the high frequency $636 \mathrm{~cm}^{-1} \mathrm{E}_{\mathrm{g}}$ mode decreases initially and then increases. From these measurements, the temperature and state of stress can be determined as a function of time. The results are shown in Fig. $4 \mathrm{c}$ and $5 \mathrm{c}$ and are addressed in the discussion section.

The temperature and state of stress of the third experiment involving laser-induced crystallization of sol-gel film deposited on the ruby interfacial layer is determined using a different procedure. The temperature is determined independently from the ruby fluorescence lifetime using the phase modulation technique. Since temperature is determined by an independent method, the Raman shift of only one of the $E_{\mathrm{g}}$ modes is required to make a determination of the transient stress in the film. Since the $E_{\mathrm{g}}$ mode at $143 \mathrm{~cm}^{-1}$ is the more intense of the two modes it was chosen. The Raman shift of this $\mathrm{g}$ mode increases during the crystallization process as is shown in Fig. 6 . 

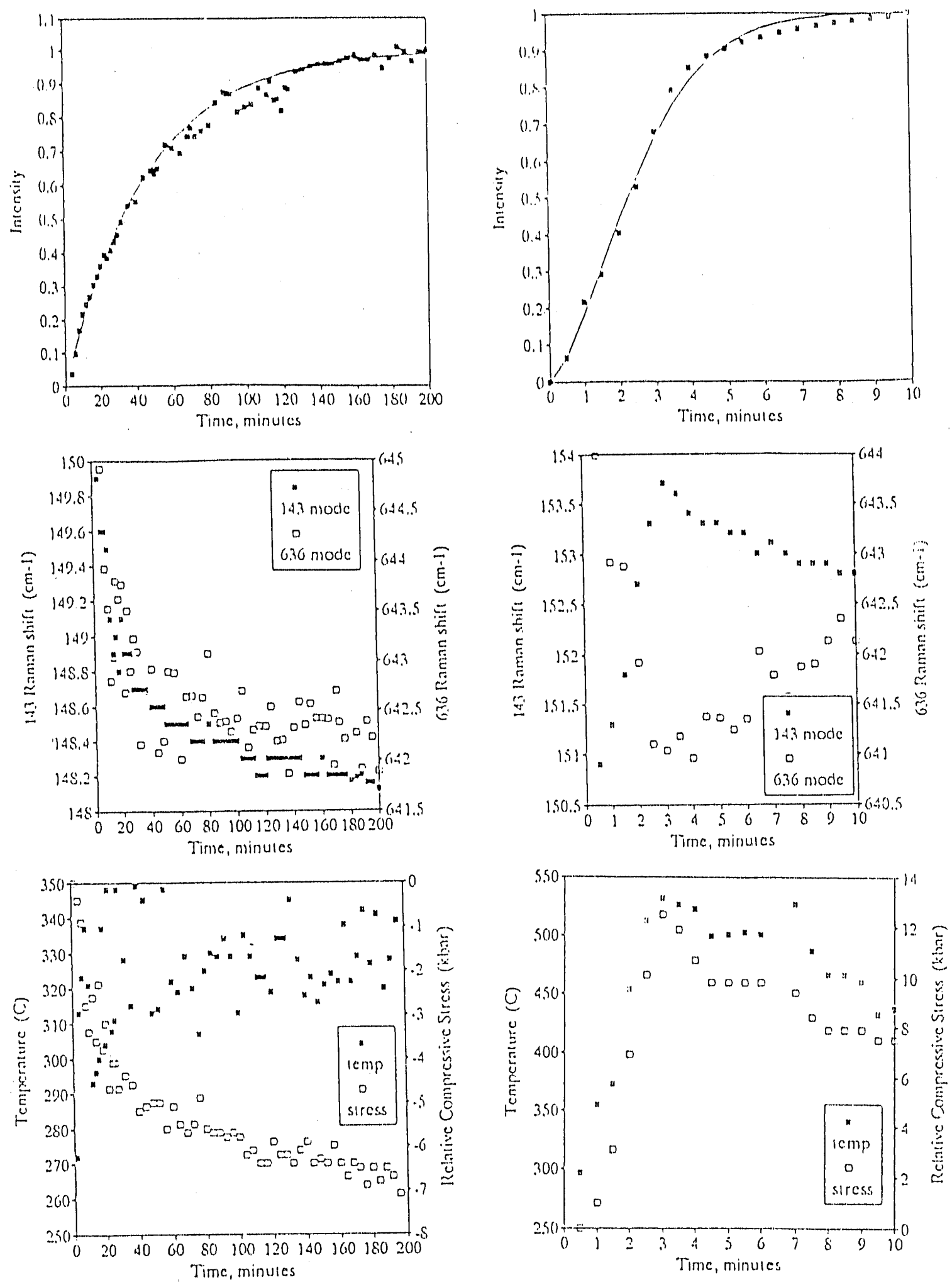

Fig.4. Isothe. mal crystallization of a frec-standing film. (a) Intensity variation with time. (b) Frequency variation with time. (c) Changes in film stress and $\mathrm{T}$ with time. 
The phase and the amplitude of the ruby fluorescence were acquired simultaneously with the Raman spectra. Changes in the phase and amplitude of the fluorescence signal are shown as a function of time in Figure 6b. The fluorescence lifetime can be determined from the phase measurement, and from the previous calibration can be correlated directly with a change in temperature. The resulting temperature and state of stress is shown as a function of time in Fig. oc.
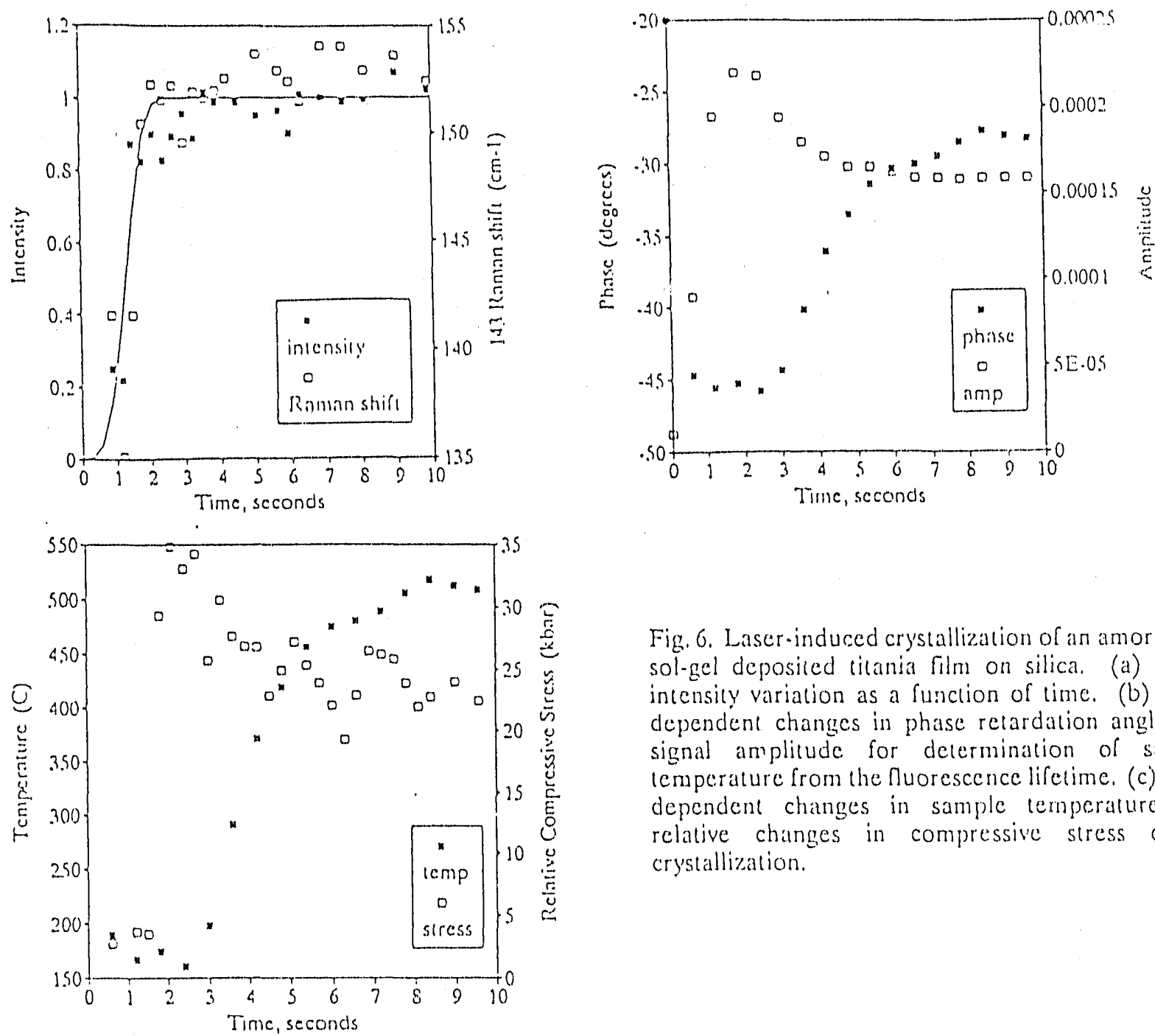

\begin{abstract}
Fig. 6. Laser-induced crystallization of an amorphous, sol-gel deposiced titania film on silica. (a) Mode incensity variation as a function of time. (b) Time: dependent changes in phase retardation angle and signal amplitude for determination of sample temperature from the nuorescence lifetime. (c) Time dependent changes in sample temperature and relative changes in compressive stress during crystallization.
\end{abstract}

\title{
5.0 DISCUSSION
}

Earlier work has demonstrated that crystallization of sol-gel deposited titania films results in 2 densification of the film, marked increase in refractive index, and a decrease in film thickness by a factor of two. [6] A spheroidal microstructure develops such that the mean diameter of the. crystallites is comparable to the film thickness. As a result, both interfacial and intergranula stresses are expected to change during evolution of the equilibrium microstructure. The rate at which this process occurs is temperature dependent so that transformations which are induced rapidly may be expected to generate a different stress state in the film than that which resuli: ai longer times under isothermal conditions. 
A number of optical methods are available for real-time non-destructive characterization of film stress and localized temperature during isothermal or laser-induced heating and subsequent crystallization of amorphous films. One lechnique for temperature determination, not discussed in this paper, involves simultaneous measurement of both Stokes and anti-Stokes Raman scattering intensities for a particular vibrational mode. The intensity ratio follow's a Boltzmann distribution from which a molecular temperature of the sample may be obtained. [13] While several experimental difficulties must be addressed, this method allows temperatures to be determined in times as short as several nanose:onds. [14] The analytical methods described in the present work involve analysis of the Stokes Raman spectrum alone or the fluorescence response of a dopant located at the film-substrate interface.

The contour method which is used to quantify both temperature and inherent stress requires simultaneous measurement of two vibrational mode frequencies. Further, the precision by which $P$ and $T$ are determined depends on the magnitudes of the quantities $\left(\partial \omega_{i} / \partial T\right)$, and $\left(\partial \omega_{i} / \partial P\right.$ ) for each of the modes in question and the precision improves as the difference in these quantitics increases. A calibration diagram also must be constructed for each frequency used from Raman measurements of well characterized samples in a heated diamond anvil cell. The fluorescence method which is a general technique for probing any material relies on the introduction of a suitable probe material at the film-substrate interface. In the case of ruby, a direct correlation between sample temperature and fluorescence lifetime has been demonstrated. For the change in relative stress observed during crystallization, there is little perturbation to this correlation by an applied stress. The measurement time is dictated by the fluorescence lifetime of the probe and the modulation frequency,. Measurements reported in this work could be made in times as short as several hundred milliseconds. Determination of interfacial stress from fluorescence wavelength measurements in ruby is somewhat more difficult as the fluorescence also exhibits a marked temperature dependence. Nevertheless it is possible to deconvolute temperature perturbations from the wavelength shift to infer the magnitude of the stress component to the wavelength shift. Since the fluorescence probe is restricted to the interface, the interfacial stress generated at the film. substrate interface can be measured directly. A difference in the computed stress state between the fluorescence and contour methods probably can be attributed to an intergranular stress present in the film. The two procedures provide a means to probe both contributions to the stress state of the film.

The optical methods discussed above were used to evaluate film temperature and residual stress during isothermal or laser-induced crystallization of amorphous titania sol-gel deposited films. During isothermal crystallization of free-standing films over a several hour time period, a decrease in compressive stress by about $7 \mathrm{kbar}$ was indicated by the data. High power CW laser irradiation of the same film induced complete crystallization in about ten minutes. Here, the compressive stress of the film first increased by about 10 bar during the first three minutes, then diminished by aboui 5 kbar during completion of the phase transformation. A similar result was observed for the film deposited over the ruby film on silica although it occurred over a much shorter time interval (10) sec). Temperature-dependent rate constants for the crystallization process are in agreement with those reported for isothermal crystallization of similar films. [6] Results for each of thes experiments suggest that following nucleation and initial growth of a crystalline phase from an amorphous matrix, compressive stress is relaxed. However, at the onset of the transformation anc during the initial stages of crystallization, a marked increase in compressive stress occurs. The magnitude of the evolving stress is sufficient to promote delamination between film and substraii which has been observed in a number of instances. 


\subsection{CONCLUSIONS}

Two non-destructive optical methods are described for evaluating transient changes in film temperature and stress during isothermal or laser-induced crystallization of amorphous sol-gel deposited titania films. The contour analysis method appears to work well for titania films probably because the two $E_{\text {g }}$ modes chosen for the analysis exhibit such contrasting behavior as a function of temperature and applied pressure. The fluorescence probe method appears to be general for all materials and is useful for evaluating film-substrate interfacial stress.

Results from measurements on free-standing films and coated silica substrates suggest that the crystallization process involves a marked increase in film compressive stress at the onset which is relaxed at longer times until the transformation is complete.

\subsection{ACKNOWLEDGEMENTS}

This work has been supported by the Materials Sciences Division of the Office of Basic Energy Sciences, U.S. Department of Energy. S Wood acknowledges support througin the NORCUS fellowship program sponsored by the U.S. Department of Energy through Contract DE-AM06-76RLO 2225. Pacific Northwest Laboratory is operated by Battelle Memorial Institute for the U.S. Department of Ene $y_{0} y^{\prime}$ under Contract DE-AC06-76-RLO 1830. The authors wish to express their appreciation to Drs. AC Hess and MI McCarthy for developing a code to analyze the frequency contour data.

\section{S.0 REFERENCES}

[1] C.Y. She, and L.S. Hsu, Probing structural properties of amorphous films with Raman spectroscopy. In NBS Special Publication 746, Ed. H.E. Bennett, A.H. Gienther, D. Milam, and B.E. Newnam, US Dept. of Commerce, Washington, DC, pp.383-394, 1988.

[2] G.J. Exarhos, and P.L. Morse, Raman studies of laser damaged single-and multi-layer opitcul coatings. Proc. Soc. Photo-Opt. Instrumen. Eng. 540:460-467, 1985.

[3] D.M. Friedrich, and G.J. Exarhos, Raman microprobe of laser-induced surface damoge regions in $\mathrm{TiO}_{2}$ and $\mathrm{ZrO}_{2}$ Coatings. In NBS Special Publization 746 , Ed. H. E. Bennett, A.. H. Guenther, D. Milam, and B.E. Newnam, US Dept. of Commerce, Washington, DC, pr. 374-382, 1988.

[4] S.M. Risser, and K.F. Ferris, Defect enhancement of local electric fields in dielectric filin. Proc. Soc. Photo-opt. Instrumen. Eng. 1441:?62-268, 1990.

[5] P.L. White, G.J. Exarhos, M. Bowden, N.M. Dixon, and D.J. Gardner, Raman microprim. studies of laser induced damage in dielectric films. J. Mater. Res. 6(1):126-133, 1991.

[6] G.J. Exarhos, and M. Aloi, Crystallite growth kinetics in isothermally annealed sol-gel films. Thin Solid Films 193/194:42-50, 1990. 
[7] G.J. Exarhos, N.J. Hess, and S. Ryan, Laser Raman measurements of dielectric coatings as a function of temperature. Proc. Soc. Photo-Opt. Instrumen. Eng. 1441:190-199, 1990.

[8] G.J. Exarhos, and N.J. Hess, Raman studies of stress-induced phase transformations in titania films. Proc. Mater. Res. Soc., Spring Meeting, Symposium J, Anaheim, CA 1991.

[9] P. Brüesch, Phonons: Theon' and Experiments I. Lattice Djnamics and Models of Interatomic Forces, Springer Series in Solid State Sciences 34, Ed. P. Fulde (Springer, Berlin), 1982.

[10] K.T.V. Grattan, R.K. Selli, and A.W. Palmer, Ruby decay'time fluorescence themometer in a fiber-optic configuration. Rev. Sci. Instrumen. 59(8):1328-1335, 1988.

[11]. K.F. Ferris, G.J. Exarhos, and C. Nguyen, Influence of solution chemistry on the microstmucture of sol-gel derived films. In NIST Special Publication 752. Ed. H.E. Bennett, A.H. Guenther, D. Milam, and B.E. Newnam, US Dept. of Commerce, Washington, DC, pp. $272-278,1986$.

[12] G.J. Exarhos, and W. M. Risen, Jr., Isothemal devitrification kinetics of sodium metaphosphate glass. J. Am. Ceram. Soc. 57(9):401-408, 1975.

[13] G.J. Exarhos, and J. W. Schaaf, High temperature Raman scattering from boron nitride coatings on resistively heated graphite surfaces. Proc. Photo-Opt. Instrumen. Eng. 1055:185193, 1989.

[14] G.J. Exarhos, and J.W. Schaaf, Raman scattering from boron nitride coaimgs at hish temperatures. J. Appl. Phys. 69(4):2543-2548, 1991. 

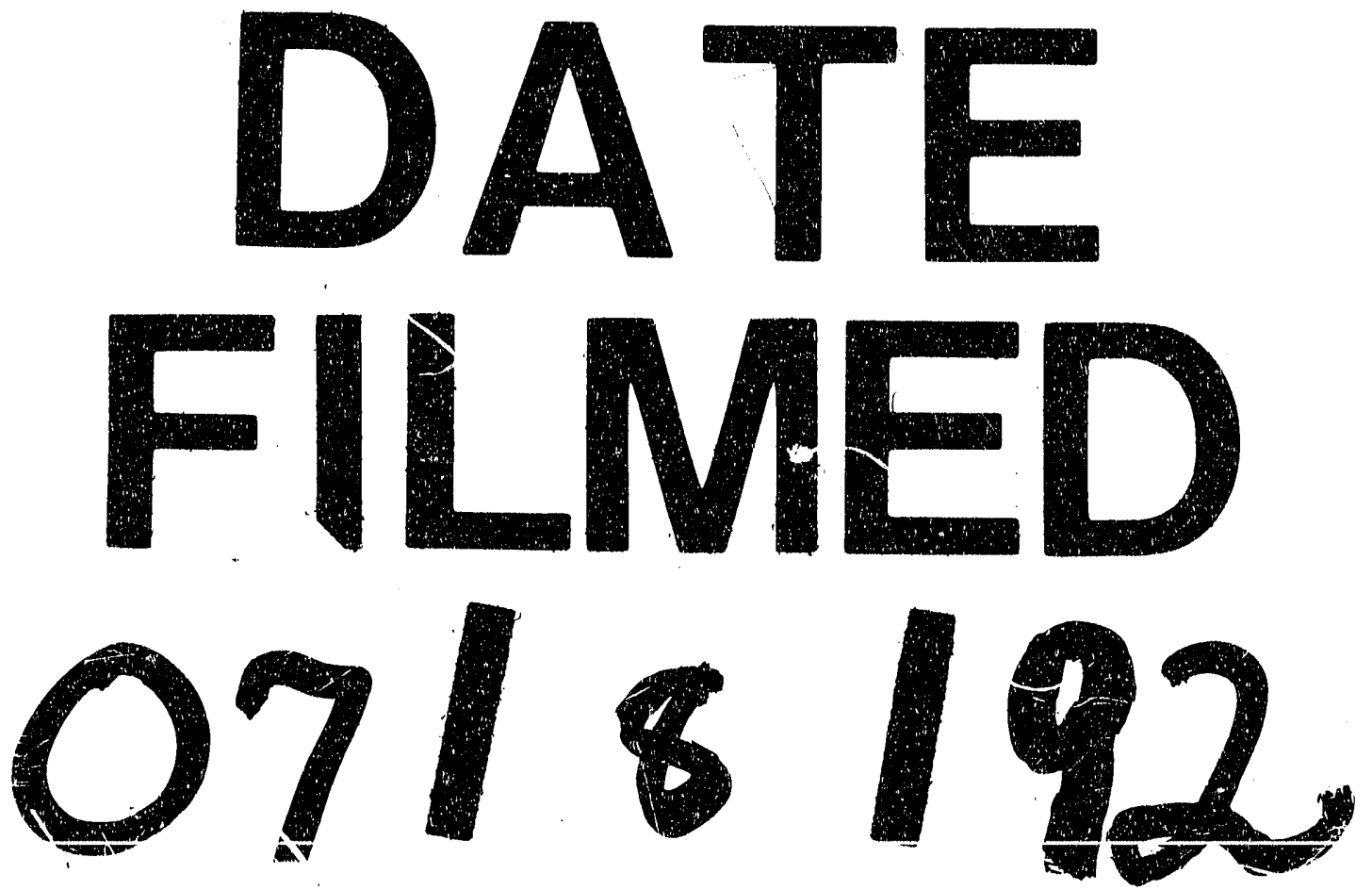


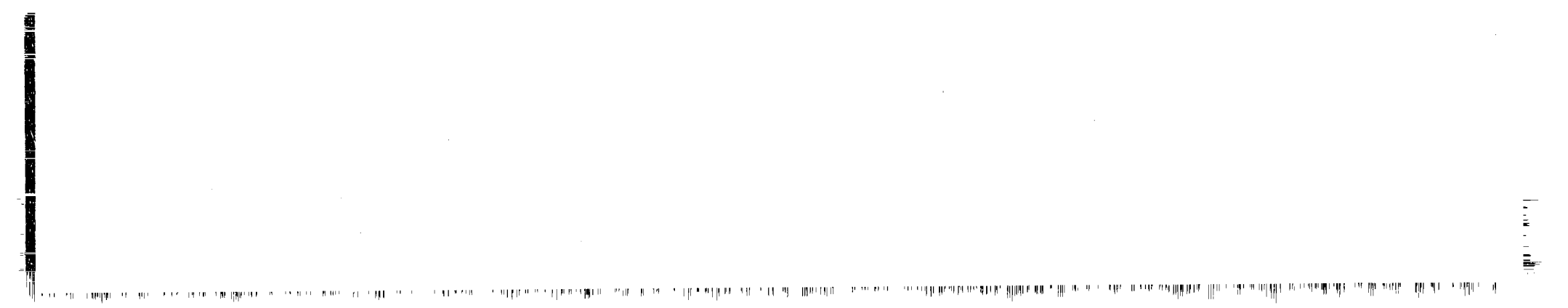

